\title{
Fixed points of multiple-valued transformations
}

by

\section{S. N. Patnaik (Montréal)}

1. Introduction. A multiple-valued transformation $F: X \rightarrow Y$ is a law assigning to each point $x$ of a topological space $X$, a closed nonempty subset $F(x)$ of a topological space $T$. A multiple-valued transformation $F: X \rightarrow Y$ is called upper semi-continuous if $F^{-1}(B)$ is closed in $X$ for each closed set $B$ in $Y$. By a multiple-valued map, it is meant an upper semi-continuous multiple-valued transformation.

A fixed point of a multiple-valued map $F: X \rightarrow X$ is a point satisfying $x_{0} \in F\left(x_{0}\right)$. The purpose of this paper is to associate a Lefschetz number [3] to a class of multiple-valued self-maps of a compact polyhedron, the non-vanishing of which implies the existence of a fixed point under the maps. Eilenberg and Montgomery [1] defined the Lefischtez number $L\left(F^{\prime}\right)$ associated with multiple-valued self-maps $F: X \rightarrow X$ of a compact polyhedron $X$ (or a metric absolute neighbourhood retract) when the image sets $F(x)$ consist of acyclic sets for all $x \in X$ and proved that $L(F) \neq 0$ implies the existence of a point $x_{0}$ satisfying $x_{0} \in F^{\prime}\left(x_{0}\right)$. Maxwell defined the Lefschetz number $L(f)$ associated with single-valued continuous maps $f: X \rightarrow X^{n} / S_{n}$ (here $X^{n} / S_{n}$ is the orbit space under the natural action of the permutation group of $n$ letters $S_{n}$ on the $n$th Cartesian product of a compact polyhedron $X$ ) and proved that $L(f) \neq 0$ implies the existence of a point $x_{0}$ such that $x_{0}$ is one of the coordinates of $f\left(x_{0}\right)$. We shall extend the method used by Maxwell in his study of fixed points of symmetric product mappings and relate it to the study of fixed points of multiplevalued maps under convenient assumptions. Our result may be regarded as an extension of the work of Maxwell [4] and that of Eilenberg and Montgomery [1] on 'the fixed points of multiple-valued maps.

It is likely that our results can be proved or generalized by a possible generalized form of the Vietoris-Begle theorem and the methods of Eilenberg and Montgomery. Some results have been obtained in these directions and will be presented in a subsequent paper.

2. Let $X$ be a compact metric space with metric $\varrho$. For two subsets $A, B$ of $X$, we denote by:

$$
d(x, A)=\inf \{\varrho(x, y) \mid y \in A\},
$$




$$
\begin{aligned}
& d(A, B)=\sup \{d(x, B) \mid x \in A\}, \\
& \delta(A, B)=\max \{d(A, B), d(B, A)\} .
\end{aligned}
$$

Here $\delta(A, B)$ is the well-known Hausdorff distance between the sets $A$ and $B$. Let $K^{n}(X)$ denote the space of all sets consisting of $n$ distinct elements of $X$. A metric is introduced in the set $K^{n}(X)$ and it becomes a topological space with the topology induced by this metric. WeImake an assumption that it is possible to introduce arbitrarily fine simplicial decompositions in the spaces $X$ and $K^{n}(X)$. The identification map $\tau: X^{n} \rightarrow K^{n}(X)$ is defined by $\tau\left(x_{1}, x_{2}, \ldots, x_{n}\right)=\left[x_{1}, x_{2}, \ldots, x_{n}\right]$ where $\left(x_{1}, x_{2}, \ldots, x_{n}\right)$ is an element of the product space $X^{n}$ and $\left[x_{1}, x_{2}, \ldots, x_{n}\right]$ is the set consisting of $n$ distinct elements of $X$ and is an element of $K^{n}(X)$. Let $X, Y$ be compact metric spaces. A continuous (single-valued) map $f: X \rightarrow Y$ induces in a natural manner $\widetilde{f}: K^{n}(X) \rightarrow K^{n}(Y)$ given by

$$
\tilde{f}\left[x_{1}, x_{2}, \ldots, x_{n}\right]=\left[f\left(x_{1}\right), f\left(x_{2}\right), \ldots, f\left(x_{n}\right)\right] .
$$

If $f_{1}, f_{2}, X \rightarrow Y$ are homotopic, then so also $\widetilde{f}_{1}, \widetilde{f}_{2},: K^{n}(X) \rightarrow K^{n}(Y)$.

Proposimion 2.1. Let $\tau: X^{n} \rightarrow K^{n}(X)$ be the identification map. Then the chain homomorphism $\tau_{\#}: C_{i}\left(X^{n}, L\right) \rightarrow C_{i}\left(K^{n}(X), L\right)$ (where $L$ is a field of coefficients) induces a homomorphism $\lambda_{\# \#}: C_{i}\left(\Pi^{n}(X), L\right) \rightarrow C_{i}\left(X^{n}, L\right)$. ( $\lambda_{\#}$ given here is similar to the transfer homomorphism given by Floyd [2]).

Proof. Let $e_{i}$ be a generator of the chain group $C_{i}\left(K^{n}(X)\right)$, then there exists an element $c_{i} \in C_{i}\left(X^{n}\right)$ with $\tau_{\#} c_{i}=e_{i}$. We define $\lambda_{\#}: O_{i}\left(K^{n}(X)\right)$ $\rightarrow C_{i}\left(X^{n}\right)$ by setting $\lambda_{\#} c_{i}=l_{i} c_{i}$ for some $l_{i} \in L$. For another generator $e_{i}^{\prime} \in C_{i}\left(K^{n}(X)\right)$, we have the equation $\lambda_{*} c_{i}^{\prime}=l_{i}^{\prime} e_{i}^{\prime}$ for some $l^{\prime} \in L$ and $c_{i}^{\prime} \in C_{i}\left(X^{n}\right)$. Now we define $\lambda_{\#}\left(e_{i}+e_{i}^{\prime}\right)=l_{i} c_{i}+l_{i}^{\prime} c_{i}^{\prime}$. It can easily be verified that $\lambda_{\#}$ thus defined on the generators of the group $C_{i}\left(K^{n}(X)\right)$ is linear and can thus be linearly extended to all elements of $C_{i}\left(K^{n}(X)\right)$. To prove that $\lambda_{\#}$ is a chain homomorphism, we need to verify that $\lambda_{\#} \partial=\partial \lambda_{\# \#}$ (where $\partial$ is the usual boundary homomorphism). Consider $\partial \lambda_{* \#} e_{i}=\partial l_{i} c_{i}=l_{i} \partial c_{i}$ $=\lambda_{\#} \partial c_{i}=\lambda_{\#} \partial \tau_{\#} c_{i}=\lambda_{\#} \tau_{\#} \partial c_{i}=\left(\lambda_{\#} \tau_{\#}\right) \partial c_{i}=l \partial c_{i}$ (we observe that $\left.\partial \tau_{\#}=\tau_{\# \#} \partial\right)$, what we have shown in above for the generators can be easily extended by linearity to all elements of $C_{i}\left(\Pi^{n}(X)\right)$.

3. Let $F$ be a multiple-valued map $F: X \rightarrow X$ where $X$ is a compact metric space; such that for each $x \in X, F^{\prime}(x)$ consists of $n$ distinct elements of $X$. The single-valued (continuous) map $f: X \rightarrow K^{n}(X)$ is defined as before by $f(x)=F(x)$ for each $x \in X$. We have the sequence of homomorphisms:

$$
\text { (3.1) } \quad C_{i}(X) \stackrel{f \# i}{\longrightarrow} C_{i}\left(K^{n}(X)\right) \stackrel{\lambda \# i}{\longrightarrow} C_{i}\left(X^{n}\right) \stackrel{\pi \# i}{\longrightarrow} C_{i}(X)
$$

where $f_{\#}$ is the usual chain homomorphism induced by the map $f, \lambda_{*}$ is the homomorphism given by the proposition 2.1 and $\pi_{\#}$ is the homomorphism induced by the projection map $\pi\left(x_{1}, x_{2}, \ldots, x_{n}\right)=x_{1}$.

On the level of homology groups (simplicial theory with a field of coefficients is used throughout), we have the sequence of homomorphisms

$$
H_{i}(X) \stackrel{\mathfrak{I}^{*} i}{\longrightarrow} H_{i}\left(K^{n}(X)\right) \stackrel{\lambda^{*} i}{\longrightarrow} H_{i}\left(X^{n}\right) \stackrel{\pi^{*} i}{\longrightarrow} H_{i}(X) .
$$

The composite homomorphism $\pi_{*_{i}} \lambda_{*_{i}} f_{*_{i}}$ is a linear transformation of the (finite dimensional) vector space $H_{i}(X)$. The Lefschetz number associated with the multiple-valued map $F$ (of the type defined earlier) is given by

$$
L\left(F^{\prime}\right)=\sum_{i}(-1)^{i} \operatorname{trace}\left(\pi_{*_{i}} \lambda_{*_{i}} f_{*_{i}}\right) .
$$

The number $L(F)$ depends only on the homotopy class of the singlevalued $\operatorname{map} f$ associated with the multiple-valued map $F$. In case $n=1$, this reduces to the well known Lefschetz number associated with a singlevalued map. This generalizes the Lefschetz number associated with a map $f: X \rightarrow X^{n} / S_{n}$ where $X^{n} / S_{n}$ is the orbit space under the action of the permutation group of $n$ letters on $X^{n}$ as defined by Maxwell [4]. Now we come to the main theorem of this paper.

THEOREM. Let $F$ be a multiple-valued map from a compact metric space $X$ into itself such that $F(x)$ consists of $n$ distinct points for each $x \in X$. Let the Lefschetz number $L(F)$ be defined as in the preceding. Then the equation $L(F) \neq 0$ implies the existence of a fixed point under $F$.

Proof. The single-valued map $f: X \rightarrow K^{n}(X)$ is defined by $f(x)=F(x)$ for each $x \in X$. The distance $\varrho(x, f(x))$ from $x$ to the nonempty closed set $f(x)$ is defined, as before, by $\varrho(x, f(x))=\inf \{\varrho(x, y) \mid y \in f(x)=F(x)\}$. We observe here that $\varrho(x, f(x))=0$ if and only if $x \in f(x)$, i.e. $x$ is fixed point under $F$.

Consider $X \stackrel{1 \times f}{\longrightarrow} \rightarrow X \times K^{n}(X) \stackrel{\psi}{\longrightarrow} R$ where maps $1 \times f$ and $\psi$ are defined by

$$
\begin{gathered}
(1 \times f)(x)=(x, f(x)), \\
\psi(x, \bar{y})=\varrho(x, \bar{y})=\inf \left\{\varrho\left(x, y_{i}\right) \mid y_{i} \in f(x)\right\}
\end{gathered}
$$

(here $\bar{y}$ denotes the set $f(x)$ ).

Suppose on the contrary there exists no fixed point under $F$, then $(1 \times f)(x)>0$ for each $x \in X$. By compactness of $X$, there exists a $\varepsilon>0$ such that

$$
\psi(1 \times f)(x)>\varepsilon \quad \text { for each } \quad x \in X .
$$

We have assumed earlier that the spaces $X$ and $K^{n}(X)$ admit simplicial decompositions and we now choose sufficiently fine triangulations such 
that mesh $X<\varepsilon / 2$ and mesh $K^{n}(X)<\varepsilon / 2$. By the simplicial approximation theorem, there exists a barycentric subdivision $X^{\prime}$ of $X$ and a simplicial map $s: X^{\prime} \rightarrow K^{n}(X)$ where $s$ is homotopic to $f$ and $\varrho(s(x), f(x))<\varepsilon / 2$.

We consider the composite of the sequence of maps

$$
X^{\prime S \cong f} \stackrel{\longrightarrow}{\longrightarrow} K^{n}(X) \stackrel{\tau^{-1}}{\longrightarrow}\left(X^{n}\right)^{\prime} \stackrel{\varphi}{\longrightarrow} X^{n} \stackrel{r}{\longrightarrow} X
$$

where $S$ is a simplicial map homotopic to $f$ which exists by the simplicial approximation theorem, $\tau^{-1}$ is the inverse of the identification map $\tau: X^{n} \rightarrow K^{n}(X)$ and is set-valued, $\varphi$ is induced by the subdivision map of the barycentric subdivision of $\left(X^{n}\right)^{\prime}$ of $X$ and $\pi$ is the projection map on the first coordinate defined by $\pi\left(x_{1}, x_{2}, \ldots, x_{n}\right)=x_{1}$. We define the setvalued map $t: X \rightarrow 2^{|X|}$ by $t(\sigma)=\pi \varphi \tau^{-1} S(|\sigma|)$ for each simplex $\sigma$ of the triangulation of $X$. It can be easily verified that $|\sigma| \cap t(|\sigma|)=\varnothing$ for each simplex $\sigma$ of the triangulation of $X$ by considering the definition of the set-valued map $t$ and the choice of the triangulations of the spaces $X$ and $K^{n}(X)$.

We now consider the sequence of chain homomorphisms

$$
C_{i}(X) \stackrel{(S d) \#_{i}}{\longrightarrow} C_{i}\left(X^{\prime}\right) \stackrel{S_{\#_{i}}}{\longrightarrow} C_{i}\left(K^{n}(X)\right) \stackrel{\pi_{i} \lambda^{2} \#_{i}}{\longrightarrow} C_{i}(X)
$$

where $(S d)_{\#}$ is the chain homomorphism induced by the map of a space $X$ into its barycentric subdivision $X^{\prime}, \pi_{\#}$ is induced by the projection map $\pi\left(x_{1}, x_{2}, \ldots, x_{n}\right)=x_{1}$ and $\lambda_{\#}$ is the transfer homomorphism as defined in proposition 2.1. Define the composite chain map $\theta_{\#_{i}}=\pi_{\#_{i}} \lambda_{\#_{i}} s_{\#_{i}}(S d)_{\#_{i}}$. This chain map $\theta_{\#}$ has no fixed elements because of $|\sigma| \cap t(|\sigma|)=\varnothing$ and by a theorem due to Lefschetz $[3], L\left(\theta_{\#}\right)=0$. Hence,

$$
\begin{aligned}
L\left(\theta_{\#}\right) & =\sum_{i}(-1)^{i} \operatorname{trace} \theta_{*_{i}} \\
& =\sum_{i}(-1)^{i} \operatorname{trace} \theta_{*_{i}} \quad \text { (by a theo } \\
& =\sum_{i}(-1)^{i} \operatorname{trace}\left(\pi_{*_{i}} \lambda_{*_{i}} s_{*_{i}}(S d)_{*_{i}}\right) \\
& =\sum_{i}(-1)^{i} \operatorname{trace}\left(\pi_{*_{i}} \lambda_{*_{i}} s_{*_{i}}\right) \\
& =\sum_{i}(-1)^{i} \operatorname{trace}\left(\pi_{*_{i}} \lambda_{*_{i}} f_{*_{i}}\right) \\
& =\sum_{i}(-1)^{i} \operatorname{trace} F_{*_{i}} \\
& =0
\end{aligned}
$$$$
=\sum_{i}(-1)^{i} \operatorname{trace} \theta_{*_{i}} \quad \text { (by a theorem due to H. Hopf) }
$$

Hence no fixed points under $F^{\prime}$ implies $L\left(F^{\prime}\right)=0$ and our proof is complete.
CoRollary. If $X$ is a acyclic polyhedron, then $L(F) \neq 0$ and every multiple-valued map $F: X \rightarrow X$ of the type discussed in the above theorem admits a fixed point.

Remark. O'Neill [5] by using a different method has obtained a similar theorem, our procedure is an extension of the work of Maxwell [4]. In the statement of the theorem the image sets $F(x)$ under $F: X \rightarrow X$ may consist of $n$ distinct acyclic components by an application of the Vietoris mapping theorem.

\section{References}

[1] S. Eilenberg and D. Montgomery, Fixed point theorems for multiple-valued transformation, Amer. J. Math. 68 (1946), pp. 214-222.

[2] E. E. Floyd, Orbit spaces for finite transformation groups I, Duke Math. J. 20 (1953), pp. 563-568.

[3] S. Lefschetz, Algebraic topology, Amer. Math. Soc. Colloq. Publications, vol. 27, Providence, R. I., 1942.

[4] C. N. Maxwell, Fixed points of symmetric product mappings, Proc. Amer. Math. Soc. 8 (1957), pp. 808-815.

[5] B. O'Neill, Induced homology homomorphisms for set valued maps, Pacific J. Math. I (1957), pp. 1179-1184.

UNIVERSITE DE MONTREAL

Montréal, Canada

Reçu par la Rédaction le 26. 3. 1968 